\title{
Identification of key target genes and pathways in laryngeal carcinoma
}

\author{
FENG LIU ${ }^{1}$, JINTAO DU $^{1}$, JUN LIU ${ }^{1}$ and BEI WEN ${ }^{2}$ \\ ${ }^{1}$ Department of Otorhinolaryngology, West China School of Medicine, West China Hospital, Sichuan University, \\ Chengdu, Sichuan $610041{ }^{2}$ Department of Otorhinolaryngology, \\ Sichuan Academy of Medical Sciences and Sichuan Provincial People's Hospital, Chengdu, Sichuan 610072, P.R. China
}

Received March 19, 2015; Accepted April 8, 2016

DOI: $10.3892 / 01.2016 .4750$

\begin{abstract}
The purpose of the present study was to screen the key genes associated with laryngeal carcinoma and to investigate the molecular mechanism of laryngeal carcinoma progression. The gene expression profile of GSE10935 [Gene Expression Omnibus (GEO) accession number], including 12 specimens from laryngeal papillomas and 12 specimens from normal laryngeal epithelia controls, was downloaded from the GEO database. Differentially expressed genes (DEGs) were screened in laryngeal papillomas compared with normal controls using Limma package in $\mathrm{R}$ language, followed by Gene Ontology (GO) enrichment analysis and pathway enrichment analysis. Furthermore, the protein-protein interaction (PPI) network of DEGs was constructed using Cytoscape software and modules were analyzed using MCODE plugin from the PPI network. Furthermore, significant biological pathway regions (sub-pathway) were identified by using iSubpathwayMiner analysis. A total of 67 DEGs were identified, including 27 up-regulated genes and 40 down-regulated genes and they were involved in different GO terms and pathways. PPI network analysis revealed that Ras association (RalGDS/AF-6) domain family member 1 (RASSF1) was a hub protein. The sub-pathway analysis identified 9 significantly enriched sub-pathways, including glycolysis/gluconeogenesis and nitrogen metabolism. Genes such as phosphoglycerate kinase 1 ( $P G K 1)$, carbonic anhydrase II (CA2), and carbonic anhydrase XII (CA12) whose node degrees were $>10$ were identified in the disease risk sub-pathway. Genes in the sub-pathway, such as RASSF1, PGK1,CA2 and CA12 were presumed to serve critical roles in laryngeal carcinoma. The present study identified DEGs and their sub-pathways in the
\end{abstract}

Correspondence to: Dr Bei Wen, Department of Otorhinolaryngology, Sichuan Academy of Medical Sciences and Sichuan Provincial People's Hospital, 32 West Second Section First Ring Road, Chengdu, Sichuan 610072, P.R. China

E-mail: beiwenbw@163.com

Key words: laryngeal cancer, differentially expressed genes, protein-protein interaction network, sub-pathway analysis disease, which may serve as potential targets for treatment of laryngeal carcinoma.

\section{Introduction}

Laryngeal cancer is one of the most common types of head and neck cancer, and the majority of laryngeal cancers are squamous cell carcinomas (1). The incidence rate of laryngeal cancer accounts for 1-5\% of all malignancies, and is third most common head and neck cancer tumor (2). Laryngeal cancer has enormous functional and psychological consequences for the patients, in particular with regard to eating and communication (3). Laryngeal papillomas which are caused primarily by human papillomavirus (HPV) types 6 and 11 (4) are the most common benign tumors in the larynx. They are associated with a 3-7\% risk of malignant transformation, in which irradiation and smoking appear to be risk factors (5). In order to further improve survival rates, diagnostic and preventive approaches, it is necessary to understand the mechanisms of laryngeal carcinogenesis.

The past decade has witnessed major progress in the understanding of the molecular alteration underlying the development of head and neck cancers (6,7). For laryngeal cancer, previous studies have revealed that the use of DNA microarray is helpful for the elucidation of laryngeal carcinogenesis $(8,9)$. Colombo et al (10) identified that the differentially expressed genes (DEGs) in larynx tumors were involved in cellular processes associated with the cancer phenotype, including the cell cycle, DNA repair, and signal transduction among which cystatin B (CSTB) encoded the protein, which has previously been associated with antimetastatic function. Ma et al (6)reported that numerous molecular abnormalities encoding for cell cycle control and integrin-mediated cell adhesion, such as matrix metallopeptidase 12 (MMP12), keratin 19 (KRT19) and proline-rich protein BstNI subfamily 1 ( $P R B 1$ ) were identified in laryngeal carcinoma cells. In addition, the findings of Oblak et al (11) demonstrated that overexpression of suppressor of cytokine signaling 1 (SOCS1) was linked with reducing the aggressive and metastatic potential in laryngeal carcinoma due to the inhibition of cancer cell proliferation. Despite the fact that considerable efforts have been made over the years, the molecular mechanisms involved in the development and 
progression of laryngeal carcinoma have not been completely demonstrated and require further investigation.

Using the same gene expression profile, DeVoti et al (12) had demonstrated that altered expression of numerous genes was associated with cellular growth and differentiation and that there was a role for altered innate immunity in recurrent respiratory papillomatosis. In the present study, microarray analysis was used to identify DEGs in growing papillomas compared with adjacent laryngeal epithelia as controls. Comprehensive bioinformatics was used to analyze the significant pathways and functions and to construct the protein-protein interaction (PPI) network in addition to significant modules to identify the critical DEGs. Furthermore, significant biological pathway regions (sub-pathways) were predicted. The present study aimed to identify involvement of genes critical in laryngeal carcinoma and to get an improved understanding of the molecular circuitry in laryngeal carcinoma.

\section{Materials and methods}

Microarray data and data preprocessing. The mRNA expression profile of GSE10935 was downloaded from the National Center of Biotechnology Information (NCBI) Gene Expression Omnibus (GEO) database (http://www.ncbi.nlm. nih.gov/geo/), which was deposited by DeVoti et al (12). The platform is GPL96 [HG-U133A] Affymetrix Human Genome U133A Array and GPL571 [HG-U133A_2] Affymetrix Human Genome U133A 2.0 Array. In this dataset, there were 12 specimens from papillomas and 12 specimens from adjacent clinically normal, laryngeal epithelia from patients with recurrent respiratory papillomatosis.

The probes were annotated with gene symbols according to the annotation information in different platforms. Gene symbols which were common across the GPL96 and GPL571 platforms were retained to the subsequent analysis. Furthermore, cross slide normalization was performed by using the surrogate variable analysis (SVA) package (13) in R (14), and within slide normalization was performed by using preprocessCore package (15) in R. The gene expression matrix of specimens was received.

Screening of DEGs. A $t$-test (16) in the limma (17) package in R (14) was used to identify DEGs. Up-regulated and down-regulated genes were identified between papillomas and normal controls. The Bonferroni method (18) was applied to perform a multiple testing correction. The threshold for the DEGs was set as corrected P-value $<0.05$ and $I \log _{2}$ fold change (FC) $\mid \geq 1$. In addition, the clustering analysis of DEGs was represented by a heat-map using gplots (19) in R (14).

Functional and pathway enrichment analysis of DEGs. In order to facilitate the functional annotation and pathway analysis, all the DEGs were analyzed using the Database for Annotation Visualization and Integrated Discovery (DAVID) (20) to perform the Gene Ontology (GO) (21) and Kyoto Encyclopedia of Genes and Genomes (KEGG) (22) analysis. The human genome was used as the background list and human was chosen as the species. The other default parameters of the tools were kept unchanged. The GO terms and KEGG pathways that were enriched by $>5$ genes and
$\mathrm{P}<0.05$ was considered to indicate a statistically significant difference.

PPI network construction and modules selection. The PPI network is represented by an undirected graph with nodes indicating the genes and edges indicating the mapped interactions of the proteins encoded by the genes (23). In the present study, all DEGs were imported into Cytoscape plugin to create network visualizations. The source of the interaction network databases were the Human Protein Reference Database (HPRD) (24) (http://www.hprd.org/) and the Biological General Repository for Interaction Datasets (BioGRID) (25) (http://thebiogrid.org/) database. Then the resulting PPI network was subjected to module analysis with the Plugin MCODE (26) with the default parameters (Degree cutoff $\geq 2$, Node score cutoff $\geq 2$, K-core $\geq 2$, and Max depth=100).

Risk sub-pathway analysis. iSubpathwayMiner (http://cran.r-project.org/web/packages/iSubpathwayMiner/) is an R platform for graph-based construction and analysis of KEGG pathways (27). For a given KEGG pathway, the sub-pathways were obtained by searching all possible paths between start-points (membrane receptors or their ligands) and end-points (transcriptional factors or their immediate targets) in the adjacency matrix generated by node relationships (28).

Disease associated risk sub-pathway creation and presentation were based on iSubpathway Miner (28). The genes which had higher degrees in the PPI network enriched in the sub-pathways were identified as the critical genes in papilloma.

\section{Results}

Data preprocessing and DEGs screening. After normalization, 22,283 probe sets mapped to 12,496 gene symbols under GPL96 platform. Total 22,277 probe sets mapped to 12,495 gene symbols under GPL571 platform. Finally, 12,495 common gene symbols were identified and the gene expression matrix of specimens was received. The gene expression profile following normalization is presented in Fig. 1. The black line in the box tends towards the same straight line, indicating a good degree of standardization.

A total of 67 DEGs were identified in papilloma compared with the normal tissues, including 27 up-regulated genes and 40 down-regulated genes. A heat-map of the DEGs is presented in Fig. 2. The results demonstrated that the DEGs expression pattern could be used to distinguish the papillomas samples from normal controls.

Functional analysis of DEGs. GO and pathway analysis indicated that up-regulated DEGs and down-regulated DEGs in papillomas samples were significantly enriched in different GO terms and KEGG pathways. A total of $17 \mathrm{GO}$ terms were enriched, for example, ectoderm development, epithelium development, epithelial cell differentiation, extracellular space, and intermediate filament cytoskeleton (Table I). In addition, 2 KEGG pathways were significantly enriched, including bladder cancer and glycolysis/gluconeogenesis.

PPI network construction and modules selection. The PPI network included 984 nodes and 1,314 interactions (Fig. 3). 

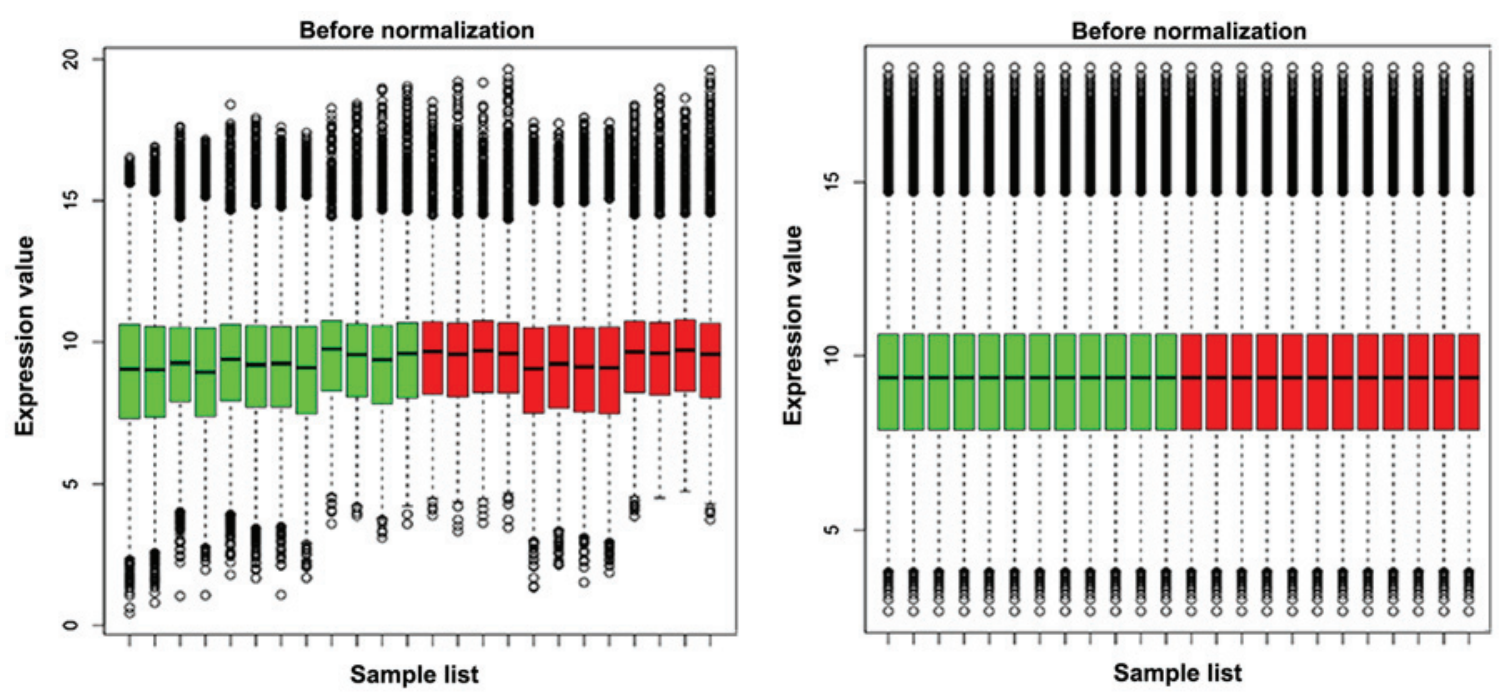

Figure 1. Normalized expressed value data. The box in the black line means the median of each set of data, which determine the degree of standardization of data through its distribution. The green box indicates the normal sample and the red box represents the laryngeal carcinoma sample.

The results demonstrated that a total of 34 genes had a node degree of $>10$, such as cyclin-dependent kinase inhibitor $1 \mathrm{~A}$ $(C D K N 1 A)($ degree $=208), \mathrm{N}$-myc downstream regulated 1 $(N D R G 1)($ degree $=87)$, lactate dehydrogenase A $(L D H A)$ $($ degree $=66)$, and Ras association $($ RalGDS/AF-6) domain family member 1 (RASSF1) (degree=48). In addition, 2 modules were identified from the PPI network (Fig. 4). Module 1 had 2 DEGs, RASSFl and inhibitor of DNA binding 1 (IDI), and another 2 genes, suppressor of variegation 3-9 homolog 1 (SUV39H1) and cell division cycle 20 (CDC20). Besides, module 2 had 2 DEGs, NDRG1 and $L D H A$, and another gene, ubiquitin $\mathrm{C}(U B C)$.

Disease risk sub-pathway analysis. A total of 9 disease risk sub-pathways were identified as presented in Table II, such as glycolysis/gluconeogenesis, nitrogen metabolism, tyrosine metabolism, and phenylalanine metabolism. It should be noted that phosphoglycerate kinase 1 ( $P G K 1)$, carbonic anhydrase II (CA2), and carbonic anhydrase XII (CA12) which were enriched in different sub-pathways including glycolysis/gluconeogenesis and nitrogen metabolism also had a higher node degree of $>10$. Moreover, aldehyde dehydrogenase 3 family, member A1 (ALDH3Al) was enriched in different pathways, including tyrosine metabolism and phenylalanine metabolism.

\section{Discussion}

Advanced laryngeal cancer is an aggressive disease, which has a low rate of treatment efficacy and a high rate of recurrence (29). An improved understanding of the pathogenesis of laryngeal cancer should open therapeutic possibilities. In the present study, DEGs in laryngeal papillomas compared with normal controls were analyzed. The analysis of DEGs revealed that the DEGs were enriched in different GO terms and pathways, such as epithelial cell differentiation and the glycolysis/gluconeogenesis pathway. Besides, RASSF 1 and IDI were enriched in module 1 that extracted from the PPI network of DEGs. Furthermore, $P G K 1, C A 2$ and $C A 12$ which had higher node degrees in the PPI network were identified to be involved in different disease risk sub-pathways, including glycolysis/gluconeogenesis and nitrogen metabolism.

RASSF1 encodes a protein similar to the RAS effector proteins and may be a potential tumor suppressor (30). Agathanggelou et al (31) have previously demonstrated that RASSF1 serves an important role in cell cycle regulation and apoptosis as well as microtubule stability and RAS-association domain family 1, isoform A gene (RASSF1A) inhibits tumor growth in both in vitro and in vivo systems. Furthermore, the findings of Sun et al (32) revealed that gene silencing through promoter hypermethylation of $\mathrm{CpG}$ islands is linked to the inactivation of tumor suppressor genes, such as RASSF1 in head and neck cancers. By contrast, ID1 is a helix-loop-helix (HLH) protein which inhibits the DNA binding and transcriptional activation ability of HLH family proteins with which it interacts (33). Coskunpinar et al (34) quantified the expression of tumorigenesis associated genes in laryngeal cancer and observed that a set of 16 genes had altered expression including IDI. Furthermore, Wang et al (35) reported that IDI could interact with CDC20 and RASSF1A during early mitosis and then led to enhanced CDC20 activity. In line with the previous study, the present study identified that RASSF1A which was a hub protein in the PPI network could interact with $I D I$ in module 1 (Fig. 4), indicating that RASSF1A may interact with IDI influencing cell cycle regulation and inhibiting tumor growth in laryngeal cancer.

In addition, the results of the present study showed that $P G K 1$ were involved in the risk sub-pathway of glycolysis/gluconeogenesis. Glycolysis has been shown to be enhanced in almost all cancers, termed the 'Warburg effect' (36). Gatenby et al (37) demonstrated that tumor cells might sustain high glycolysis not only for biosynthetic precursor production and energy, but also for the eradication of adjacent normal cells within organ parenchyma. Besides, PGK1 is a glycolytic enzyme and may also act as a polymerase alpha cofactor (primer recognition protein) (38). Ning et al (39) reported that $P G K 1$ in the glycolysis/gluconeogenesis pathway was significantly differentially expressed between laser micro-dissected malignant compared 

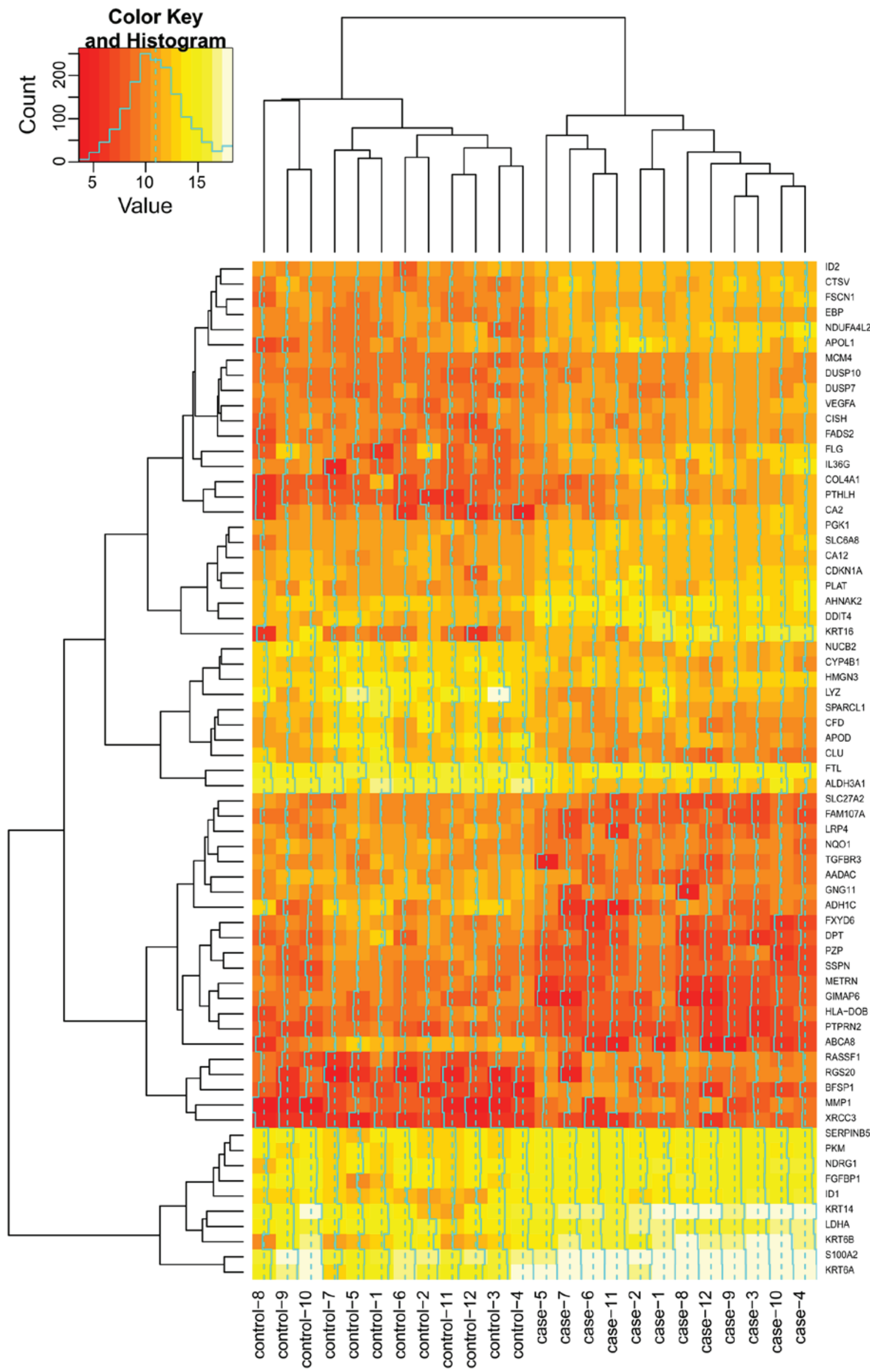

Figure 2. Heat-map overview of the differentially expressed genes. Brightness level indicates the gene expression value. When the gene expression value is higher, the color is brighter. 
Table I. The enriched gene ontology terms of differentially expressed genes.

\begin{tabular}{|c|c|c|c|}
\hline Category & Term & P-value & Genes \\
\hline $\mathrm{BP}$ & $\begin{array}{l}\text { Ectoderm } \\
\text { development }\end{array}$ & $1.21 \mathrm{E}-04$ & $\begin{array}{l}\text { PTHLH, KRT6A, KRT6B, KRT16, } \\
\text { FLG, KRT14, AHNAK2 }\end{array}$ \\
\hline BP & $\begin{array}{l}\text { Epithelium } \\
\text { development }\end{array}$ & 0.001897 & $\begin{array}{l}\text { PTHLH, FLG, VEGFA, KRT14, } \\
\text { AHNAK2, CA2 }\end{array}$ \\
\hline BP & $\begin{array}{l}\text { Epithelial cell } \\
\text { differentiation }\end{array}$ & 0.002007 & $\begin{array}{l}\text { PTHLH, FLG, VEGFA, KRT14, } \\
\text { AHNAK2 }\end{array}$ \\
\hline BP & $\begin{array}{l}\text { Epidermis } \\
\text { development }\end{array}$ & 0.005772 & $\begin{array}{l}\text { PTHLH, KRT16, FLG, KRT14, } \\
\text { AHNAK2 }\end{array}$ \\
\hline BP & $\begin{array}{l}\text { Regulation of cell } \\
\text { proliferation }\end{array}$ & 0.010959 & $\begin{array}{l}\text { PTHLH, CDKN1A, KRT6A, ID2, } \\
\text { VEGFA, CLU, TGFBR3, FGFBP1, DPT }\end{array}$ \\
\hline $\mathrm{BP}$ & $\begin{array}{l}\text { Positive regulation of } \\
\text { cell proliferation }\end{array}$ & 0.037925 & $\begin{array}{l}\text { LDHA, ADH1C, FADS2, NQO1, } \\
\text { ALDH3A1, CYP4B1, FTL }\end{array}$ \\
\hline $\mathrm{BP}$ & Oxidation reduction & 0.037925 & $\begin{array}{l}\text { LDHA, ADH1C, FADS2, NQO1, } \\
\text { ALDH3A1, CYP4B1, FTL }\end{array}$ \\
\hline $\mathrm{BP}$ & $\begin{array}{l}\text { Negative regulation of } \\
\text { molecular function }\end{array}$ & 0.041489 & PTHLH, CDKN1A, ID2, ID1, NQO1 \\
\hline $\mathrm{CC}$ & Extracellular region part & 2.57E-09 & $\begin{array}{l}\text { PLAT, PZP, COL4A1, SPARCL1, CLU, } \\
\text { LYZ, FXYD6, MMP1, PTHLH, } \\
\text { METRN, APOL1, APOD, SERPINB5, } \\
\text { VEGFA, NUCB2, TGFBR3, CA2, CFD, } \\
\text { FGFBP1, DPT }\end{array}$ \\
\hline $\mathrm{CC}$ & Extracellular space & $5.20 \mathrm{E}-08$ & $\begin{array}{l}\text { PLAT, PZP, CLU, LYZ, FXYD6, PTHLH, } \\
\text { METRN, APOL1, APOD, SERPINB5, } \\
\text { VEGFA, NUCB2, TGFBR3, CA2, CFD, } \\
\text { FGFBP1 }\end{array}$ \\
\hline $\mathrm{CC}$ & Extracellular region & $2.02 \mathrm{E}-04$ & $\begin{array}{l}\text { PLAT, PZP, COL4A1, SPARCL1, CLU, } \\
\text { LYZ, FXYD6, MMP1, PTHLH, } \\
\text { METRN, APOL1, APOD, SERPINB5, } \\
\text { VEGFA, NUCB2, TGFBR3, CA2, CFD, } \\
\text { FGFBP1, DPT }\end{array}$ \\
\hline $\mathrm{CC}$ & Intermediate filament & $8.58 \mathrm{E}-04$ & $\begin{array}{l}\text { KRT6A, KRT6B, KRT16, FLG, } \\
\text { KRT14, BFSP1 }\end{array}$ \\
\hline $\mathrm{CC}$ & $\begin{array}{l}\text { Intermediate filament } \\
\text { cytoskeleton }\end{array}$ & $9.46 \mathrm{E}-04$ & $\begin{array}{l}\text { KRT6A, KRT6B, KRT16, FLG, KRT14, } \\
\text { BFSP1 }\end{array}$ \\
\hline $\mathrm{CC}$ & Endoplasmic reticulum & 0.038809 & $\begin{array}{l}\text { AADAC, EBP, NUCB2, TGFBR3, } \\
\text { FADS2, SLC27A2, HLA-DOB, } \\
\text { ALDH3A1, CYP4B1 }\end{array}$ \\
\hline $\mathrm{CC}$ & $\begin{array}{l}\text { Proteinaceous extracellular } \\
\text { matrix }\end{array}$ & 0.040784 & $\begin{array}{l}\text { COL4A1, SPARCL1, VEGFA, MMP1, } \\
\text { DPT }\end{array}$ \\
\hline MF & $\begin{array}{l}\text { Structural constituent of } \\
\text { cytoskeleton }\end{array}$ & $2.31 \mathrm{E}-04$ & $\begin{array}{l}\text { KRT6A, KRT6B, KRT16, KRT14, } \\
\text { BFSP1 }\end{array}$ \\
\hline MF & $\begin{array}{l}\text { Structural molecule } \\
\text { activity }\end{array}$ & 0.043459 & $\begin{array}{l}\text { KRT6A, KRT6B, COL4A1, KRT16, } \\
\text { FLG, KRT14, BFSP1 }\end{array}$ \\
\hline
\end{tabular}

$\mathrm{BP}$, biological process, $\mathrm{CC}$, cellular component, MF, molecular function.

with benign clinical samples of prostate tissue. Moreover, Sun et al (32) observed that PGK1 were up-regulated in the tumor xenograft that originated from transketolase-like 1 (TKTL1) -expressing O11 cells which was from head and neck squamous cell carcinoma. Thus, $P G K 1$ may serve an essential role in the glycolysis/gluconeogenesis pathway in promoting laryngeal cancer progression.
Furthermore, these findings revealed that $C A 2$ and $C A 12$ which had higher node degrees in PPI network were associated with nitrogen metabolism sub-pathway. Glutamine is the major source of nitrogen metabolism for nucleotide and amino acid synthesis, although many cells can metabolize glutamine in excess of their nitrogen requirement (40). Tennant et al (41) demonstrated that glutamine was an essential nutrient for 


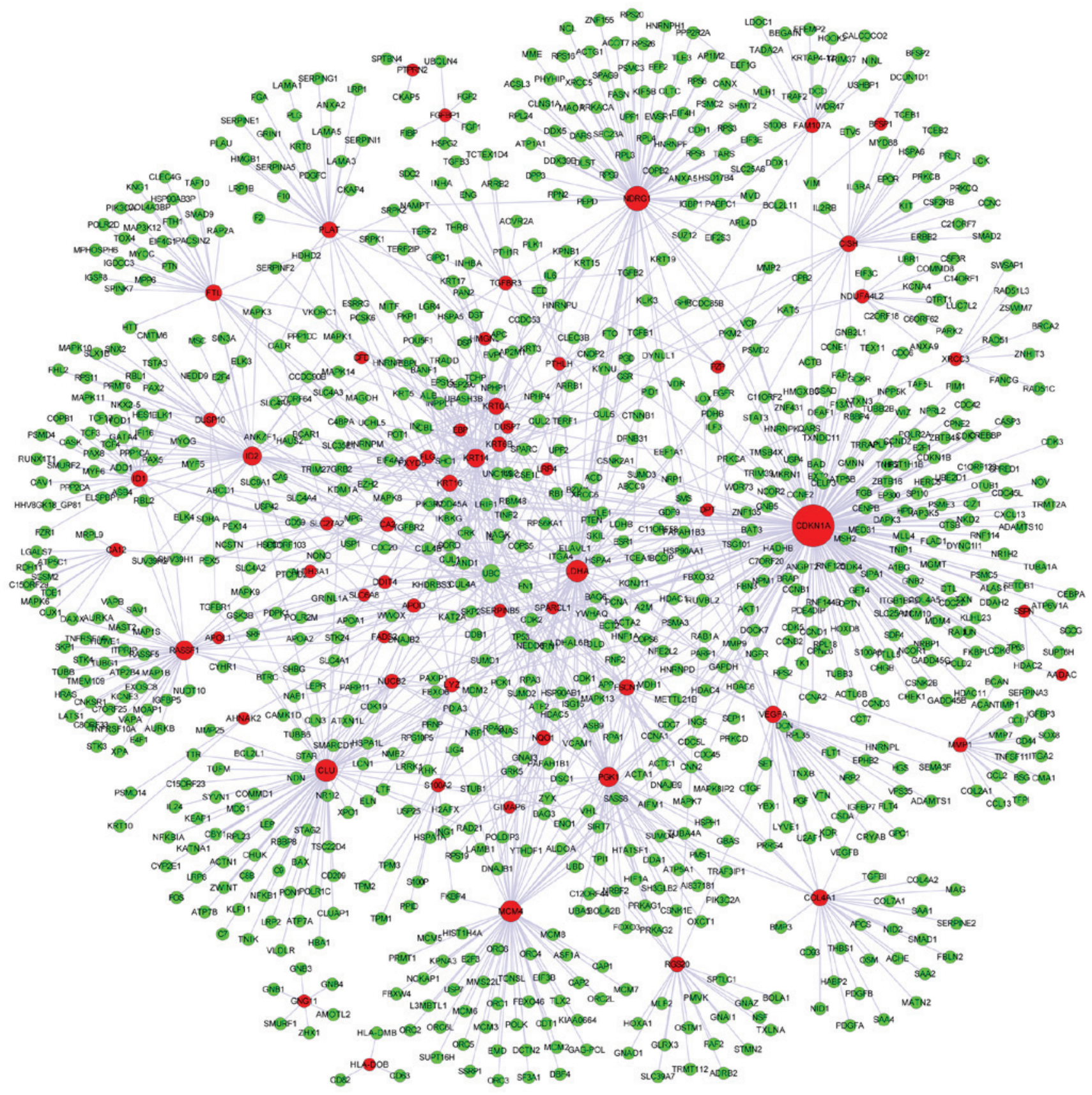

Figure 3. Protein-protein interaction network of DEGs. The red nodes indicate DEGs and green nodes represent genes not differentially expressed. DEGs, differentially expressed genes.

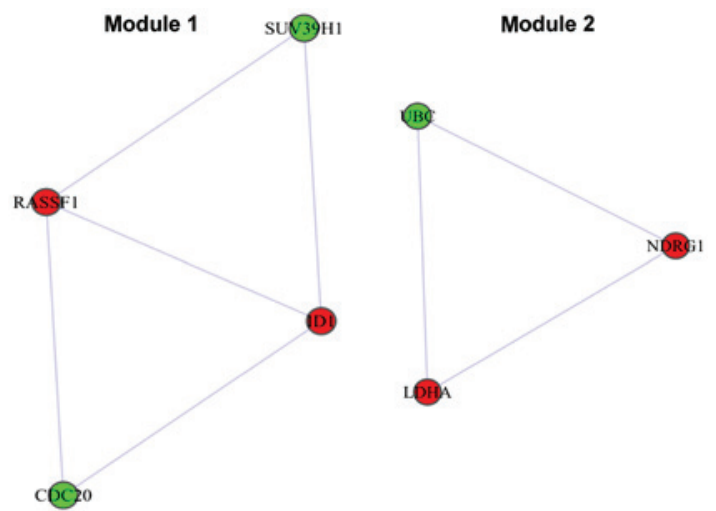

Figure 4. Two highly connected modules extracted from the protein-protein interaction network. many tumor cells and glutamine depletion may contribute to the effectiveness of the drug in acute lymphoblastic leukaemia. By contrast, CA2 and CA12 are two of several isozymes of carbonic anhydrase which can catalyze reversible hydration of carbon dioxide (42). Dasgupta et al (43) discovered that $C A 2$ overexpressed in head and neck cancer cell line. In addition, Parkkila et al (44) showed that transmembrane CA12 were highly expressed in certain tumors, and may be implicated in the acidification of the extracellular enviroment surrounding cancer cells, thus creating a microenvironment beneficial to tumor growth and spread. Besides, Lascorz et al (45) found that $C A 2$ and $C A 12$ were enriched in the KEGG pathway nitrogen metabolism and the GO category carbonate dehydratase activity in microarray studies 
Table II. The statistically significant sub-pathways identified of differentially expressed genes.

\begin{tabular}{llr}
\hline Pathway & \multicolumn{1}{c}{ Genes } & FDR \\
\hline Glycolysis/Gluconeogenesis & PGK1, PKM, ALDH3A1 & 0.000937 \\
Nitrogen metabolism & CA2, CA12 & 0.005907 \\
Metabolism of xenobiotics by cytochrome P450 & CYP4B1, ALDH3A1 & 0.005907 \\
Steroid biosynthesis & EBP & 0.027276 \\
Tyrosine metabolism & ALDH3A1 & 0.027276 \\
Phenylalanine metabolism & ALDH3A1 & 0.040641 \\
Drug metabolism-cytochrome P450 & ALDH3A1, CYP4B1 & 0.040641 \\
Fatty acid metabolism & SLC27A2 & 0.040641 \\
Histidine metabolism & ALDH3A1 & 0.042535
\end{tabular}

FDR, represents false discovery rate.

on colorectal carcinogenesis. In this context, $C A 2$ and $C A 12$ may serve crucial roles in nitrogen metabolism beneficial to laryngeal cancer progression.

In summary, the present study identified several key genes (RASSF1, PGK1, CA2 and CA12) that participated in different pathways (glycolysis/gluconeogenesis, and nitrogen metabolism) were involved in the mechanism of laryngeal cancer. RASSF1A may inhibit tumor growth in laryngeal cancer via interacting with $I D I$ involving in the cell cycle regulation. Besides, PGK1 may serve an essential role in the glycolysis/gluconeogenesis pathway in promoting laryngeal cancer progression. In addition, $C A 2$ and $C A 12$ may be crucial for nitrogen metabolism beneficial to laryngeal cancer progression. These efforts may provide an improved understanding of molecular mechanisms underlying the carcinogenesis of laryngeal cancer. Further investigation of the genes identified in this study and prospective studies to determine their potential clinical value are needed.

\section{Acknowledgements}

The present study was granted by National Basic Research Program of China grants 2002 (grant no. 0040205401078).

\section{References}

1. Licitra L, Bernier J, Grandi C, Locati L, Merlano M, Gatta G and Lefebvre JL: Cancer of the larynx. Crit Rev Oncol Hematol 47: 65-80, 2003.

2. Chen H, Zhou L, Dou T, Wan G, Tang. H and Tian J: BMI1'S maintenance of the proliferative capacity of laryngeal cancer stem cells. Head Neck 33: 1115-1125, 2011.

3. Ferlito A, Rogers SN, Shaha AR, Bradley PJ and Rinaldo A: Quality of life in head and neck cancer. Acta Otolaryngol 123: 5-7, 2003.

4. Torrente MC, Rodrigo JP, Haigentz M Jr, Dikkers FG, Rinaldo A, Takes RP, Olofsson J and Ferlito A: Human papillomavirus infections in laryngeal cancer. Head Neck 33: 581-586, 2011.

5. Hobbs CG and Birchall MA: Human papillomavirus infection in the etiology of laryngeal carcinoma. Curr Opin Otolaryngol Head Neck Surg 12: 88-92, 2004.

6. Ma LJ,Li W, Zhang X, Huang DH, Zhang H, Xiao JY and Tian YQ: Differential gene expression profiling of laryngeal squamous cell carcinoma by laser capture microdissection and complementary DNA microarrays. Arch Med Res 40: 114-123, 2009.

7. Nakanishi H, Taccioli C, Palatini J,Fernandez-Cymering C, Cui R, Kim T, Volinia S and Croce C: Loss of miR-125b-1 contributes to head and neck cancer development by dysregulating TACSTD2 and MAPK pathway. Oncogene 33: 702-712, 2014.
8. Järvinen A, Autio R, Haapa-Paananen S, Wolf M, Saarela M, Grénman R, Leivo I, Kallioniemi O, Mäkitie AA and Monni O: Identification of target genes in laryngeal squamous cell carcinoma by high-resolution copy number and gene expression microarray analyses. Oncogene 25: 6997-7008, 2006.

9. Fountzilas E, Markou K, Vlachtsis K, Nikolaou A, Arapantoni-Dadioti P, Ntoula E, Tassopoulos G, Bobos M, Konstantinopoulos P, Fountzilas G and Spentzos D: Identification and validation of gene expression models that predict clinical outcome in patients with early-stage laryngeal cancer. Ann Oncol 23: 2146-2153, 2012.

10. Colombo J, Fachel AA, De Freitas Calmon M, Cury PM, Fukuyama EE, Tajara EH, Cordeiro JA, Verjovski-Almeida S, Reis EM and Rahal P: Gene expression profiling reveals molecular marker candidates of laryngeal squamous cell carcinoma. Oncol Rep 21: 649-663, 2009.

11. Oblak A and Jerala R: Toll-like receptor 4 activation in cancer progression and therapy. Clin Dev Immunol 2011: 609-579, 2011.

12. DeVoti JA, Rosenthal DW, Wu R, Abramson AL, Steinberg BM and Bonagura VR: Immune dysregulation and tumor-associated gene changes in recurrent respiratory papillomatosis: A paired microarray analysis. Mol Med 14: 608-617, 2008.

13. Parker HS, Leek JT, Favorov AV, Considine M, Xia X, Chavan S, Chung $\mathrm{CH}$ and Fertig EJ: Preserving biological heterogeneity with a permuted surrogate variable analysis for genomics batch correction. Bioinformatics 30: 2757-2763, 2014.

14. Ihaka R and Gentleman R: R: A language for data analysis and graphics. J Comput Graph Stat 5: 299-314, 1996.

15. Yuan K, Liang W and Zhang J: A comprehensive analysis of differentially expressed genes and pathways in abdominal aortic aneurysm. Mol Med Rep 12: 2707-2714, 2015.

16. Cui $X$ and Churchill GA: Statistical tests for differential expression in cDNA microarray experiments. Genome Biol 4: 210, 2003.

17. Diboun I, Wernisch L, Orengo CA and Koltzenburg M: Microarray analysis after RNA amplification can detect pronounced differences in gene expression using limma. BMC Genomics 7: 252, 2006.

18. Bland JM and Altman DG: Multiple significance tests: The Bonferroni method. BMJ 310: 170, 1995.

19. Belda-Ferre P, Alcaraz LD, Cabrera-Rubio R, et al: The oral metagenome in health and disease. ISME J 6: 46-56, 2012.

20. Huang da W, Sherman BT and Lempicki RA: Systematic and integrative analysis of large gene lists using DAVID bioinformatics resources. Nat Protoc 4: 44-57, 2009.

21. Ashburner M, Ball CA, Blake JA, Botstein D, Butler H, Cherry JM, Davis AP, Dolinski K, Dwight SS, Eppig JT, et al: Gene ontology: Tool for the unification of biology. The Gene Ontology Consortium. Nat Genet 25: 25-29, 2000.

22. Kanehisa M and Goto S: KEGG: Kyoto encyclopedia of genes and genomes. Nucleic Acids Res 28: 27-30, 2000.

23. Köhler S, Bauer S, Horn D and Robinson PN: Walking the interactome for prioritization of candidate disease genes. Am J Hum Genet 82: 949-958, 2008.

24. Keshava Prasad TS, Goel R, Kandasamy K, Keerthikumar S, Kumar S, Mathivanan S, Telikicherla D, Raju R, Shafreen B, Venugopal A, et al: Human protein reference database-2009 update. Nucleic Acids Res 37 (Database Issue): D767-D772, 2009. 
25. Stark C, Breitkreutz BJ, Chatr-Aryamontri A, Boucher L, Oughtred R, Livstone MS, Nixon J, Van Auken K, Wang X, Shi X, et al: The BioGRID interaction database: 2011 update. Nucleic Acids Res 39 (Database Issue): D698-D704, 2011.

26. Bandettini WP, Kellman P, Mancini C, Booker OJ, Vasu S, Leung SW, Wilson JR, Shanbhag SM, Chen MY and Arai AE: MultiContrast delayed enhancement (MCODE) improves detection of subendocardial myocardial infarction by late gadolinium enhancement cardiovascular magnetic resonance: A clinical validation study. J Cardiovasc Magn Reson 14: 83, 2012.

27. Li C and Li MC: Package 'iSubpathwayMiner', 2013.

28. Chen X, Xu J, Huang B, Li J, Wu X, Ma L, Jia X, Bian X, Tan F, Liu L, et al: A sub-pathway-based approach for identifying drug response principal network. Bioinformatics 27: 649-654, 2011.

29. Ayaz L, Görür A, Yaroğlu HY, Özcan C and Tamer L: Differential expression of microRNAs in plasma of patients with laryngeal squamous cell carcinoma: Potential early-detection markers for laryngeal squamous cell carcinoma. J Cancer Res Clin Oncol 139: 1499-1506, 2013.

30. Tommasi S, Dammann R, Jin SG, Zhang XF, Avruch J and Pfeifer GP: RASSF3 and NORE1: Identification and cloning of two human homologues of the putative tumor suppressor gene RASSF1. Oncogene 21: 2713-2720, 2002.

31. Agathanggelou A, Cooper WN and Latif F: Role of the Ras-association domain family 1 tumor suppressor gene in human cancers. Cancer Res 65: 3497-3508, 2005.

32. Sun W, Liu Y, Glazer CA, Shao C, Bhan S, Demokan S, Zhao M, Rudek MA, Ha PK and Califano JA: TKTL1 is activated by promoter hypomethylation and contributes to head and neck squamous cell carcinoma carcinogenesis through increased aerobic glycolysis and HIF1alpha stabilization. Clin Cancer Res 16: 857-866, 2010.

33. Sakurai D, Yamaguchi A, Tsuchiya N, Yamamoto $K$ and Tokunaga K: Expression of ID family genes in the synovia from patients with rheumatoid arthritis. Biochem Biophys Res Commun 284: 436-442, 2001.

34. Coskunpinar E, Oltulu YM, Orhan KS, Tiryakioglu NO, Kanliada D and Akbas F: Identification of a differential expression signature associated with tumorigenesis and metastasis of laryngeal carcinoma. Gene 534: 183-188, 2014.
35. Wang X, Di K, Zhang X, Han HY, Wong YC, Leung SC and Ling MT: Id-1 promotes chromosomal instability through modification of $\mathrm{APC} / \mathrm{C}$ activity during mitosis in response to microtubule disruption. Oncogene 27: 4456-4466, 2008.

36. Bi X, Lin Q, Foo TW, Joshi S, You T, Shen HM, Ong CN, Cheah PY, Eu KW and Hew CL: Proteomic analysis of colorectal cancer reveals alterations in metabolic pathways: Mechanism of tumorigenesis. Mol Cell Proteomics 5: 1119-1130, 2006.

37. Gatenby RA and Gillies RJ: Why do cancers have high aerobic glycolysis? Nat Rev Cancer 4: 891-899, 2004

38. Valentini G, Maggi M and Pey AL: Protein stability, folding and misfolding in human PGK1 deficiency. Biomolecules 3: 1030-1052, 2013

39. Ning QY, Wu JZ, Zang N, Liang J, Hu YL and Mo ZN: Key pathways involved in prostate cancer based on gene set enrichment analysis and meta analysis. Genet Mol Res 10: 3856-3887, 2011.

40. Vander Heiden MG: Targeting cancer metabolism: A therapeutic window opens. Nat Rev Drug Discov 10: 671-684, 2011

41. Tennant DA, Durán RV and Gottlieb E: Targeting metabolic transformation for cancer therapy. Nat Rev Cancer 10: 267-277, 2010.

42. Lindskog S: Structure and mechanism of carbonic anhydrase. Pharmacol Ther 74: 1-20, 1997.

43. Dasgupta S, Tripathi PK, Qin H, Bhattacharya-Chatterjee M, Valentino $J$ and Chatterjee SK: Identification of molecular targets for immunotherapy of patients with head and neck squamous cell carcinoma. Oral Oncol 42: 306-316, 2006.

44. Parkkila S, Rajaniemi H, Parkkila AK, Kivela J, Waheed A, Pastorekova S, Pastorek J and Sly WS: Carbonic anhydrase inhibitor suppresses invasion of renal cancer cells in vitro. Proc Natl Acad Sci USA 97: 2220-2224, 2000.

45. Lascorz J, Hemminki K and Försti A: Systematic enrichment analysis of gene expression profiling studies identifies consensus pathways implicated in colorectal cancer development. J Carcinog 10: 7, 2011. 